of the carcinomatic serum there was always found less cystine than in the normal one. The ratio of the cystine concentration agrees with the ratio of the polarographic 'waves' of the denatured or proteolysed serum proteins (see Fig. 1).

These results were controlled by a colorimetric estimation of cystine in the hydrolysates using the Folin-Looney uric acid reagent as modified by Mirsky and Anson ${ }^{4}$. Thus in carcinomatic serum the content of the protein cystine was found to be smaller than in the normal serum.

As to the question whether the group responsible for the polarographic reaction is - $-\mathrm{S}-\mathrm{S}-$ or - $-\mathrm{SH}$, polarographic as well as colorimetric tests show that the sulphydryl groups are not present in an appreciable amount, so that the effect must be mainly due to the disulphidic groups.

Details of this research will soon be published elsewhere.

Physico-chemical Institute, Charles' University, Prague. April 16.

${ }^{1}$ Nature 139, 330 (1937).

2 Mirsky, A. E., and Anson, M. L., J. Gen. Physiol., 19, 427 and 439 (1936).

${ }^{3}$ Brdicka, R., Collection, 5, 238 (1933).

- Mirsky, A. B., and Anson, M. L., J. Gen. Physiol., 18, 307 (1935).

\section{Sign of the Magnetic Moment of Free Neutrons}

Using a method recently described by us ${ }^{1}$, we have now succeeded in determining the sign of the magnetic moment of free neutrons.

The method is based on the fact that the direction of precession of particles in a magnetic field depends on the relative direction of spin and magnetic moment (this is the meaning of the term "sign of the magnetic moment"). The direction of precession can be observed by using bars of magnetized iron as polarizer and analyser ${ }^{2}$, which are placed at an angle relative to each other ${ }^{1,3}$.

The experimental arrangement was essentially the same as before ${ }^{1}$ except that one of the iron rings was rotated through an angle of $90^{\circ}$ with respect to the other, with the neutron beam as axis, making the polarizing and analysing fields perpendicular to each other and to the neutron beam.

A short solenoid was placed between polarizer and analyser, coaxial with the neutron beam. The field in the solenoid (which was found to be fairly homogeneous, on account of the iron below and above) was adjusted so that the neutrons should rotate by $90^{\circ}$, on the average, on passing through the coil. (For calculating this field the magnetic moment of the neutrons was assumed to be 2 N.M.). The neutrons leaving the polarizer in the 'parallel' position arrived at the analyser in the 'parallel' or 'antiparallel' position, depending on the direction of precession, with correspondingly different probabilities of getting through.

The current in the solenoid was reversed at short intervals, and 480,000 counts in all were taken. An unambiguous difference of $(1 \cdot 10 \pm 0 \cdot 29)$ per cent of the total intensity (of which about 50 per cent was due to thermal neutrons) was found on reversing the current. The magnitude of the difference was of the order expected, which shows that the field in the solenoid was correctly chosen.

From the sign of the difference we conclude that the magnetic moment of the free neutron is negative that is, the relative direction of spin and magnetic moment is the same as in the electron. This agrees with expectations based on the magnetic moments of proton and deuteron.

Institute of

Theoretical Physics, Copenhagen. May 12.

1 NATURe, 139, 756 (1937).

2 Bloch, F., Phys. Rev., 50, 259 (1936).

${ }^{3}$ A somewhat similar method has been proposed by I. I. Rabi, Phys. Rev., 51, 652 (1937). It may be well to point out that the ulassical concept of precession gives the same result, in our case, as the quantum mechanical calculation.

\section{Experimental Test of the Proton-Neutron Exchange Interaction}

IT has already been pointed out ${ }^{1}$ that, when a proton or neutron of relativistic velocities traverses matter, it travels until it makes a head-on collision with a neutron or proton respectively in the material, transferring nearly its whole energy to this particle, which then travels forward in very nearly the same direction. This process is repeated again, the latter particle transferring nearly its whole energy to a proton if it be a neutron, and vice versa. Let $q$ denote the effective cross-section for either of these processes. We thus get a chain, the proton giving place to a neutron, which then gives place to a proton and so on. This effect is characteristic of an exchange interaction between a proton and a neutron, and would not occur with a non-exchange interaction. As it is important to know the form the protonneutron interaction takes in the relativistic case, we wish to point out in this note that there is an experiment which could show if the effect mentioned above does or does not exist.

Suppose we have three counters in a vertical plane, $d_{1}$ and $d_{2}$ being the distances between 1 and 2 and 2 and 3 respectively. Let us suppose that a proton passes through the topmost counter $\mathrm{I}$ in the right direction to pass through the other counters. It can then be shown easily that the chance of a proton passing through the counter 2 is

$$
\left(\sigma_{p}+\sigma_{n} e^{-\sigma q d_{1}}\right) / \sigma,
$$

where $\sigma_{p}$ and $\sigma_{n}$ are the number of protons and neutrons per c.c. of the substance respectively, and $\sigma \equiv \sigma_{p}+\sigma_{n}$. The chance of the counters 1, 2 and 3 being traversed simultaneously by protons is then

$$
\left(\sigma_{p}+\sigma_{n} e^{-\sigma q d d_{1}}\right)\left(\sigma p+\sigma^{n} e^{-\sigma q d_{2}}\right) / \sigma^{2},
$$

and the chance of protons going through 1 and 3 irrespective of whether a proton or neutron traverses 2 is

$$
\left\{\sigma_{p}+\sigma_{n} e^{-\sigma q\left(d_{1}+d_{2}\right)}\right\} / \sigma .
$$

The ratio of triple coincidences in $1,2,3$ to double coincidence between 1 and 3 is therefore

$R\left(d_{1}, d_{2}\right) \equiv \frac{\left(\sigma_{p}+\sigma_{n} e^{-\sigma q d_{1}}\right)\left(\sigma_{p}+\sigma_{n} e^{-\sigma q d_{2}}\right)}{\sigma\left\{\sigma_{p}+\sigma_{n} e^{-\sigma q\left(d_{1}+d_{2}\right)}\right\}}$

If

$$
\sigma q d_{1} \gg 1, \quad \sigma q d_{2} \gg 1,
$$

then the above ratio is $\sigma_{p} /\left(\sigma_{p}+\sigma_{n}\right)$, and if either $d_{1}$ or $d_{2}$ is zero it is unity, as is otherwise obvious. Thus 